\title{
Electronic Dance Music Launchpad Composer Android Application
}

\author{
Nur Hadisukmana*, Ida Made Santika Kusuma Yogi \\ President University \\ ${ }^{\star}$ Corresponding author: nurhadisukmana@gmail.com
}

\begin{abstract}
Music is the art of combining vocal or instrumental sounds or tones in varying melody, harmony, rhythm, and timbre. Music can come from any instruments, even there are many musician use household furniture to create a music. Nowadays, the development of music industry is much influenced by technology. One new genre music that emerge because of technology development is EDM. EDM is stand for Electronic Dance Music. EDM is a broad range of percussive electronic music genres produced largely for nightclubs, raves and festival. A lot of software, applications and tools were created to compose the sound to create EDM. Although these software are easy to install but most of these software are difficult to use and take time to learn it. What people need in this era are simplicity, portability, user friendly and easy to use.
\end{abstract}

\section{INTRODUCTION}

From the past until now music is part of human life and cannot be separated. Music is composed and performed for many purposes, ranging from aesthetic pleasure, religious or ceremonial purposes, or as an entertainment. Todays, there are so many kind of innovations of music instrument both complex and simple. Example of the simple one is instrument made from comb. This instrument found by young men from Indonesia. The sound is like saxophone. DJ sets and mixer to create electronic music are the example of complex music instrument.

Electronic music or most familiar called EDM is one of top popular genre right now. People who create or compose this music genre usually called as DJ. The most famous DJ right now are Hardwell, Steve Aoki and David Gueta. With their high creativity they can combine and mix any beat of sound to become good EDM.

To be able to compose music like them certainly need a complete set of equipment and this equipment is certainly not cheap. The advance of gadget todays allows us to develop the tools in application form.

\section{LIMITATIONS}

This application has several limitations, which are:

1. Application only work in Android OS 5.0 (Lollipop) or above.
2. The Record Music feature cannot record only the internal audio.

\section{APPLICATION PREVIEW}

This section is giving the application preview of its implementation. This section will include the use case and some application screenshots. Figure 3.1 will shows the use case diagram of the application.

Figure 3.1 Use Case Diagram

Figure 3.2 is the main screen if user open

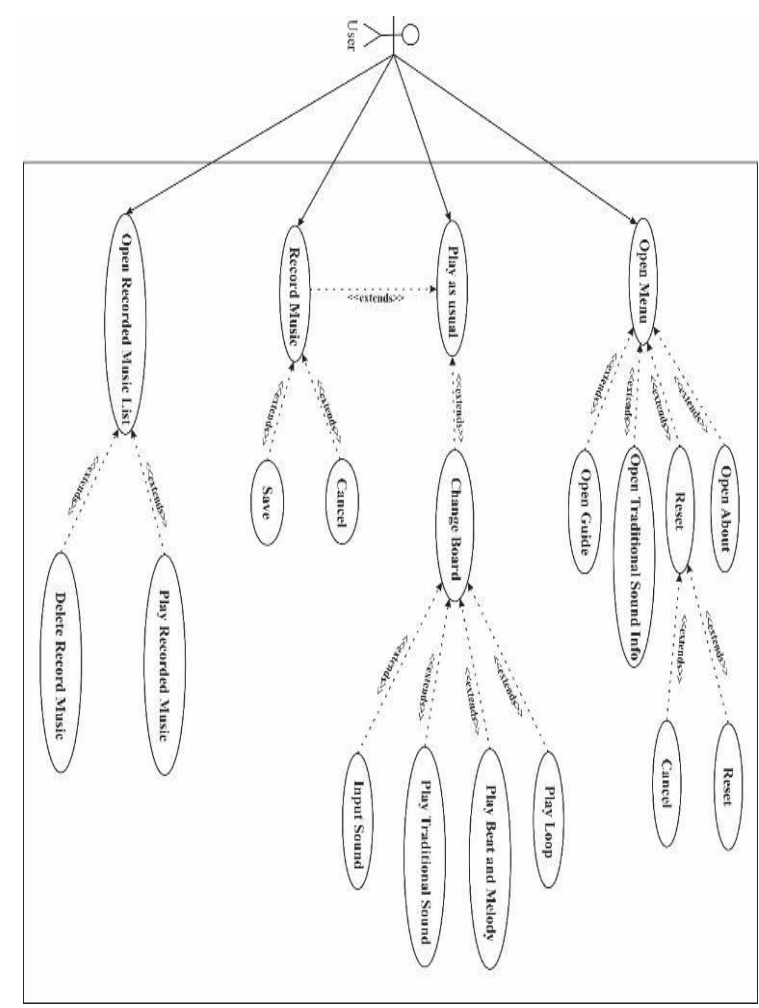

the application. If user open the recorded music then Figure 3.3 is the interface. Figure 3.4 is the interface of save record process. Figure 3.5 is the menu list interface. Figure $3.6-3.9$ are the interface of each sub menu. 


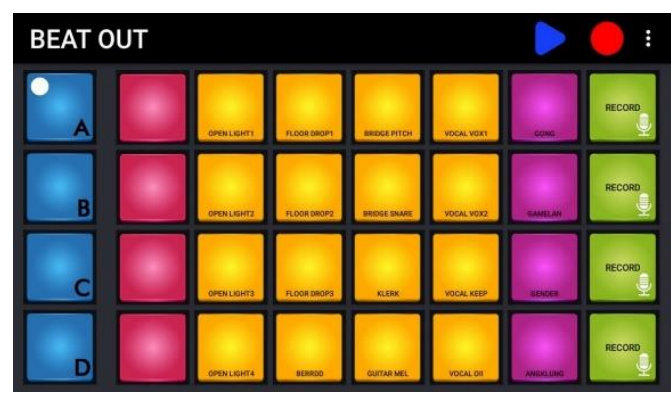

Figure 3.2 Main Screen

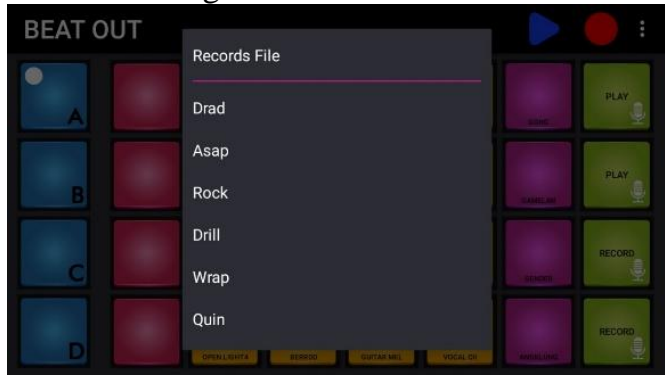

Figure 3.3 Open Recorded Music List

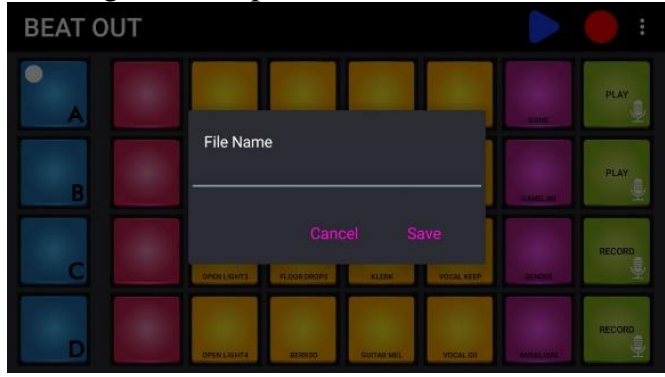

Figure 3.4 Save Record Dialog Box

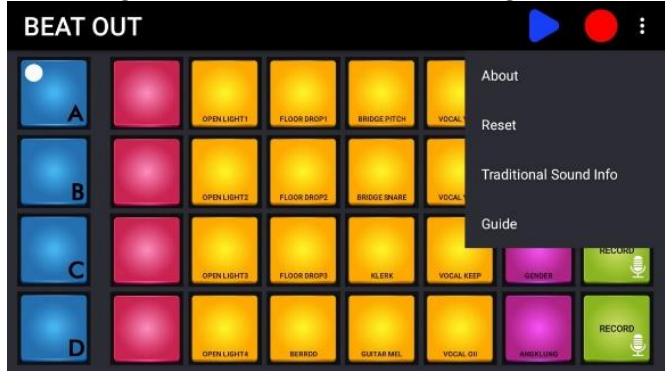

Figure $3.5 \mathrm{Menu}$

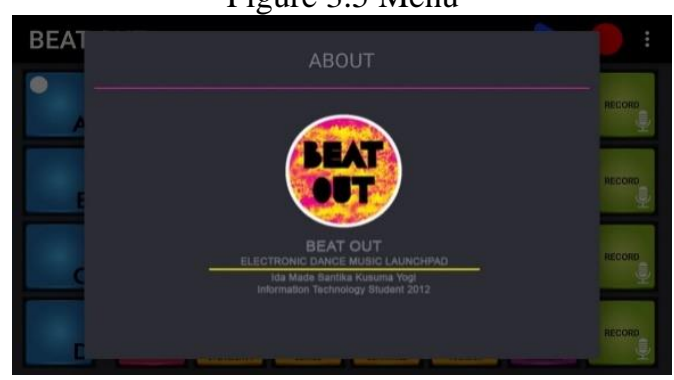

Figure 3.6 About Beat Out

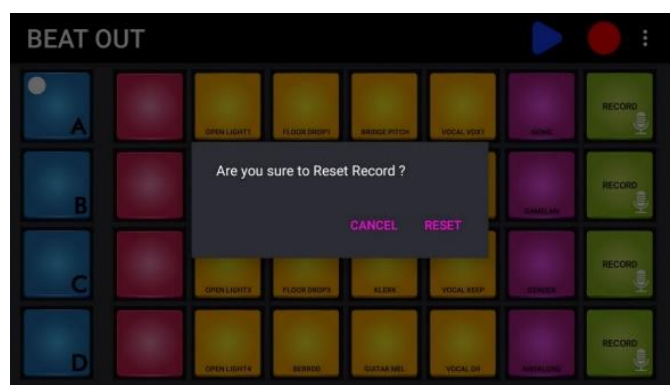

Figure 3.7 Reset

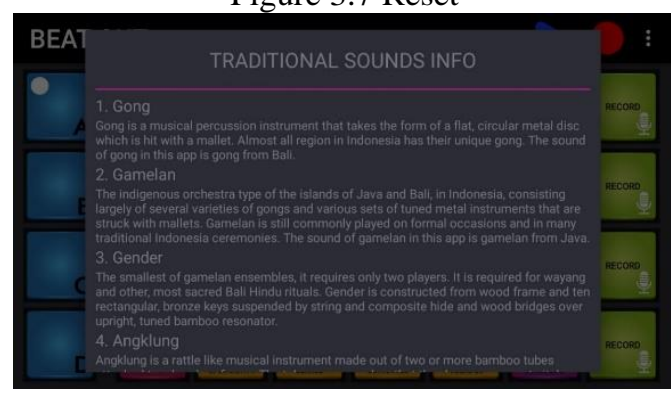

Figure 3.8 Traditional Sound Info

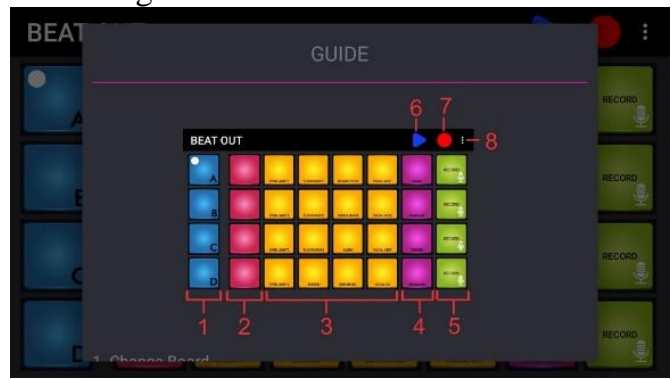

Figure 3.9 Guide

IV. EXPERIMENTAL RESULT

In order to check and be sure that application run properly, some simulations are needed to check it. Simulation will be divided based on the activities of the program executed. Testing scenarios also will show and check if the application work properly according to the expectation.

\section{- Main Screen Handling Section}

Main screen is the the page that opened when user open the application.

\begin{tabular}{|c|c|c|c|c|}
\hline $\begin{array}{l}\mathrm{N} \\
\mathrm{O}\end{array}$ & $\begin{array}{c}\text { Case } \\
\text { Scenario }\end{array}$ & $\begin{array}{l}\text { Case } \\
\text { Detail }\end{array}$ & $\begin{array}{c}\text { Expected } \\
\text { Result }\end{array}$ & Result \\
\hline 1 & $\begin{array}{l}\text { Start } \\
\text { Applicatio } \\
\mathrm{n}\end{array}$ & $\begin{array}{l}\text { Click on } \\
\text { the } \\
\text { applicatio } \\
\mathrm{n} \text { icon in } \\
\text { the } \\
\text { device }\end{array}$ & $\begin{array}{l}\text { System } \\
\text { start the } \\
\text { applicatio } \\
\mathrm{n} \text { and } \\
\text { display } \\
\text { the } \\
\text { Splash } \\
\text { Screen } \\
\text { for } \\
\text { several } \\
\text { seconds }\end{array}$ & $\begin{array}{l}\text { As } \\
\text { expecte } \\
\text { d }\end{array}$ \\
\hline
\end{tabular}


IT FOR SOCIETY, Vol. 03, No. 02

ISSN 2503-2224

\begin{tabular}{|c|c|c|c|c|}
\hline & & & $\begin{array}{l}\text { and then } \\
\text { proceed } \\
\text { to the } \\
\text { Main } \\
\text { screen. }\end{array}$ & \\
\hline 2 & $\begin{array}{l}\text { Displayin } \\
\text { g Main } \\
\text { Screen }\end{array}$ & $\begin{array}{l}\text { Main } \\
\text { Screen } \\
\text { will } \\
\text { displayed } \\
\text { after the } \\
\text { Splash } \\
\text { Screen } \\
\text { done }\end{array}$ & $\begin{array}{l}\text { Loaded } \\
\text { the main } \\
\text { screen } \\
\text { which } \\
\text { consist of } \\
8 \text { features } \\
\text { namely } \\
\text { Change } \\
\text { board } \\
\text { buttons, } \\
\text { looping } \\
\text { sound } \\
\text { buttons, } \\
\text { beat and } \\
\text { melody } \\
\text { buttons, } \\
\text { traditiona } \\
1 \text { sound } \\
\text { buttons, } \\
\text { input } \\
\text { sound } \\
\text { button, } \\
\text { save } \\
\text { record } \\
\text { list } \\
\text { button, } \\
\text { record } \\
\text { music } \\
\text { button } \\
\text { and menu } \\
\text { button. }\end{array}$ & $\begin{array}{l}\text { As } \\
\text { expecte } \\
\text { d }\end{array}$ \\
\hline
\end{tabular}

- Menu Handling Section

This test is used to make sure the Menu work proplerly.

\begin{tabular}{|c|l|l|l|l|}
\hline $\begin{array}{c}\mathrm{N} \\
\mathrm{o}\end{array}$ & $\begin{array}{l}\text { Case } \\
\text { Scenario }\end{array}$ & \multicolumn{1}{|c|}{$\begin{array}{c}\text { Case } \\
\text { Detail }\end{array}$} & $\begin{array}{l}\text { Expected } \\
\text { Result }\end{array}$ & Result \\
\hline 1 & $\begin{array}{l}\text { Displayin } \\
\text { g the list } \\
\text { Menu in } \\
\text { popup } \\
\text { interface }\end{array}$ & $\begin{array}{l}\text { Click on } \\
\text { the Menu } \\
\text { button in } \\
\text { Main } \\
\text { Screen }\end{array}$ & $\begin{array}{l}\text { The list } \\
\text { of menu } \\
\text { will show } \\
\text { in dialog } \\
\text { popup } \\
\text { interface }\end{array}$ & $\begin{array}{l}\text { As } \\
\text { expecte } \\
\text { d }\end{array}$ \\
\hline 2 & $\begin{array}{l}\text { Displayin } \\
\text { g the } \\
\text { About } \\
\text { Beat Out } \\
\text { sub menu }\end{array}$ & $\begin{array}{l}\text { Click } \\
\text { "About" } \\
\text { text in } \\
\text { menu list }\end{array}$ & $\begin{array}{l}\text { About } \\
\text { Beat Out } \\
\text { menu is } \\
\text { loaded }\end{array}$ & $\begin{array}{l}\text { As } \\
\text { expecte } \\
\text { d }\end{array}$ \\
\hline 3 & $\begin{array}{l}\text { Reset sub } \\
\text { Menu }\end{array}$ & $\begin{array}{l}\text { Click } \\
\text { "Reset" } \\
\text { text in } \\
\text { menu list }\end{array}$ & $\begin{array}{l}\text { Reset } \\
\text { alert } \\
\text { dialog is } \\
\text { loaded }\end{array}$ & $\begin{array}{l}\text { As } \\
\text { expecte } \\
\text { d }\end{array}$ \\
\hline 4 & $\begin{array}{l}\text { Displayin } \\
\text { g the } \\
\text { Tradition }\end{array}$ & $\begin{array}{l}\text { Click } \\
\text { "Tradition } \\
\text { al Sound }\end{array}$ & $\begin{array}{l}\text { Tradition } \\
\text { al Sound } \\
\text { Info }\end{array}$ & $\begin{array}{l}\text { As is } \\
\text { expecte } \\
\text { d }\end{array}$ \\
\hline
\end{tabular}

\begin{tabular}{|c|l|l|l|l|}
\hline & $\begin{array}{l}\text { al Sound } \\
\text { Info sub } \\
\text { menu }\end{array}$ & $\begin{array}{l}\text { Info" text } \\
\text { in menu } \\
\text { list }\end{array}$ & loaded & \\
\hline 5 & $\begin{array}{l}\text { Displayin } \\
\text { g the } \\
\text { Guide } \\
\text { sub menu }\end{array}$ & $\begin{array}{l}\text { Click } \\
\text { "Guide" } \\
\text { text in } \\
\text { menu list }\end{array}$ & $\begin{array}{l}\text { Guide } \\
\text { menu is } \\
\text { loaded }\end{array}$ & $\begin{array}{l}\text { As } \\
\text { expecte } \\
\text { d }\end{array}$ \\
\hline
\end{tabular}

- Looping Sound Handling Section

\begin{tabular}{|c|c|c|c|c|}
\hline $\begin{array}{c}\mathrm{N} \\
\mathrm{O}\end{array}$ & $\begin{array}{c}\text { Case } \\
\text { Scenari } \\
\text { o }\end{array}$ & $\begin{array}{l}\text { Case } \\
\text { Detail }\end{array}$ & $\begin{array}{c}\text { Expected } \\
\text { Result }\end{array}$ & Result \\
\hline 1 & $\begin{array}{l}\text { Turn on } \\
\text { the } \\
\text { looping } \\
\text { sound }\end{array}$ & $\begin{array}{l}\text { Click } \\
\text { one of } \\
\text { loop } \\
\text { button }\end{array}$ & $\begin{array}{l}\text { Sound loop } \\
\text { start playing }\end{array}$ & $\begin{array}{l}\text { As } \\
\text { expecte } \\
\text { d }\end{array}$ \\
\hline 2 & $\begin{array}{l}\text { Turn off } \\
\text { the } \\
\text { looping } \\
\text { sound }\end{array}$ & $\begin{array}{l}\text { Click } \\
\text { again } \\
\text { the } \\
\text { loop } \\
\text { button } \\
\text { that on } \\
\text { before } \\
\text { to } \\
\text { make } \\
\text { it off }\end{array}$ & $\begin{array}{l}\text { Sound loop } \\
\text { stop playing }\end{array}$ & $\begin{array}{l}\text { As } \\
\text { expecte } \\
\text { d }\end{array}$ \\
\hline 3 & $\begin{array}{l}\text { Turn on } \\
\text { the first } \\
\text { loop } \\
\text { sound } \\
\text { then } \\
\text { turn on } \\
\text { another } \\
\text { loop } \\
\text { sound }\end{array}$ & $\begin{array}{l}\text { Click } \\
\text { one of } \\
\text { loop } \\
\text { button } \\
\text { to On } \\
\text { it, then } \\
\text { click } \\
\text { anothe } \\
\text { r loop } \\
\text { button } \\
\text { to On } \\
\text { it also. }\end{array}$ & $\begin{array}{l}\text { First Sound } \\
\text { loop start } \\
\text { playing. } \\
\text { When } \\
\text { another } \\
\text { Sound loop is } \\
\text { on then the } \\
\text { first Sound } \\
\text { loop is stop } \\
\text { automatically } \\
\text {. }\end{array}$ & $\begin{array}{l}\text { As } \\
\text { expecte } \\
\text { d }\end{array}$ \\
\hline
\end{tabular}

- Beat and Melody Handling Section

\begin{tabular}{|c|l|l|l|l|}
\hline $\begin{array}{c}\text { N } \\
\text { o }\end{array}$ & $\begin{array}{c}\text { Case } \\
\text { Scenario }\end{array}$ & $\begin{array}{c}\text { Case } \\
\text { Detail }\end{array}$ & $\begin{array}{l}\text { Expected } \\
\text { Result }\end{array}$ & Result \\
\hline 1 & $\begin{array}{l}\text { Turn on } \\
\text { the beat } \\
\text { and } \\
\text { Melody }\end{array}$ & $\begin{array}{l}\text { Click the } \\
\text { beat and } \\
\text { melody } \\
\text { button }\end{array}$ & $\begin{array}{l}\text { Beat and } \\
\text { melody } \\
\text { sound } \\
\text { start } \\
\text { playing }\end{array}$ & $\begin{array}{l}\text { As } \\
\text { expecte } \\
\mathrm{d}\end{array}$ \\
\hline 2 & $\begin{array}{l}\text { Maximu } \\
\text { m beat } \\
\text { and } \\
\text { melody } \\
\text { sound } \\
\text { playing } \\
\text { in the } \\
\text { same } \\
\text { time is 3 }\end{array}$ & $\begin{array}{l}\text { Click 3 or } \\
\text { more } \\
\text { buttons of } \\
\text { beat and } \\
\text { melody } \\
\text { sound in } \\
\text { the same } \\
\text { time or } \\
\text { play } \\
\text { maximu } \\
\text { m 3 beat } \\
\text { and } \\
\text { melody } \\
\text { sound. }\end{array}$ & $\begin{array}{l}\text { As } \\
\text { expecte } \\
\text { d }\end{array}$ \\
\end{tabular}

- Traditional Sound Handling Section

\begin{tabular}{|l|l|l|l|l|}
$\mathrm{N}$ & Case & Case & Expected & Result \\
\hline
\end{tabular}


IT FOR SOCIETY, Vol. 03, No. 02

ISSN 2503-2224

\begin{tabular}{|c|c|c|c|c|}
\hline $\mathrm{O}$ & Scenario & Detail & Result & \\
\hline 1 & $\begin{array}{l}\text { Turn On } \\
\text { the } \\
\text { Tradition } \\
\text { al Sound }\end{array}$ & $\begin{array}{l}\text { Click the } \\
\text { tradition } \\
\text { al button }\end{array}$ & $\begin{array}{l}\text { Traditional } \\
\text { Sound start } \\
\text { playing }\end{array}$ & $\begin{array}{l}\text { As } \\
\text { expecte } \\
\text { d }\end{array}$ \\
\hline 2 & $\begin{array}{l}\text { Turn On } \\
\text { another } \\
\text { Tradition } \\
\text { al Sound } \\
\text { when } \\
\text { there is a } \\
\text { Tradition } \\
\text { al sound } \\
\text { still } \\
\text { playing }\end{array}$ & $\begin{array}{l}\text { Click } \\
\text { another } \\
\text { Tradition } \\
\text { al Sound } \\
\text { button }\end{array}$ & $\begin{array}{l}\text { It will } \\
\text { playing the } \\
\text { last } \\
\text { Traditional } \\
\text { Sound } \\
\text { button that } \\
\text { clicked. } \\
\text { The old } \\
\text { Traditional } \\
\text { Sound will } \\
\text { automatical } \\
\text { ly stopped. }\end{array}$ & $\begin{array}{l}\text { As } \\
\text { expecte } \\
\text { d }\end{array}$ \\
\hline
\end{tabular}

- Input Sound Handling Section

\begin{tabular}{|c|c|c|c|c|}
\hline $\begin{array}{l}N \\
\mathrm{O}\end{array}$ & $\begin{array}{c}\text { Case } \\
\text { Scenari } \\
\text { o }\end{array}$ & $\begin{array}{c}\text { Case } \\
\text { Detail }\end{array}$ & $\begin{array}{c}\text { Expected } \\
\text { Result }\end{array}$ & Result \\
\hline 1 & $\begin{array}{l}\text { Turn On } \\
\text { the } \\
\text { input } \\
\text { sound }\end{array}$ & $\begin{array}{l}\text { Click the } \\
\text { input } \\
\text { button } \\
\text { which } \\
\text { has } \\
\text { "Record } \\
\text { "text on } \\
\text { button }\end{array}$ & $\begin{array}{l}\text { Input } \\
\text { sound is } \\
\text { started by } \\
\text { record } \\
\text { using } \\
\text { microphon } \\
\text { e }\end{array}$ & $\begin{array}{l}\text { As } \\
\text { expecte } \\
\text { d }\end{array}$ \\
\hline 2 & $\begin{array}{l}\text { Turn } \\
\text { Off the } \\
\text { input } \\
\text { sound }\end{array}$ & $\begin{array}{l}\text { Click the } \\
\text { input } \\
\text { button } \\
\text { which } \\
\text { has } \\
\text { "End" } \\
\text { text on } \\
\text { button }\end{array}$ & $\begin{array}{l}\text { Input } \\
\text { sound is } \\
\text { stopped } \\
\text { and change } \\
\text { the text on } \\
\text { button } \\
\text { become } \\
\text { "Play" }\end{array}$ & $\begin{array}{l}\text { As } \\
\text { expecte } \\
\text { d }\end{array}$ \\
\hline 3 & $\begin{array}{l}\text { Play the } \\
\text { input } \\
\text { sound }\end{array}$ & $\begin{array}{l}\text { Click the } \\
\text { input } \\
\text { button } \\
\text { which } \\
\text { has } \\
\text { "Play" } \\
\text { text on } \\
\text { button }\end{array}$ & $\begin{array}{l}\text { The input } \\
\text { sound start } \\
\text { playing }\end{array}$ & $\begin{array}{l}\text { As } \\
\text { expecte } \\
\text { d }\end{array}$ \\
\hline
\end{tabular}

- Save Record Handling Section

\begin{tabular}{|c|l|l|l|l|}
\hline No & $\begin{array}{c}\text { Case } \\
\text { Scenario }\end{array}$ & $\begin{array}{l}\text { Case } \\
\text { Detail }\end{array}$ & $\begin{array}{l}\text { Expected } \\
\text { Result }\end{array}$ & Result \\
\hline 1 & $\begin{array}{l}\text { Turn On } \\
\text { the Save } \\
\text { Record } \\
\text { Music to } \\
\text { start the } \\
\text { recording }\end{array}$ & $\begin{array}{l}\text { Click } \\
\text { the } \\
\text { start } \\
\text { button }\end{array}$ & $\begin{array}{l}\text { The } \\
\text { system } \\
\text { start } \\
\text { recording }\end{array}$ & $\begin{array}{l}\text { As } \\
\text { expected }\end{array}$ \\
\hline 2 & $\begin{array}{l}\text { Turn Off } \\
\text { the Save } \\
\text { Record } \\
\text { Music to }\end{array}$ & $\begin{array}{l}\text { Click } \\
\text { the } \\
\text { stop } \\
\text { button }\end{array}$ & $\begin{array}{l}\text { The } \\
\text { system } \\
\text { stop } \\
\text { recording }\end{array}$ & $\begin{array}{l}\text { As } \\
\text { expected }\end{array}$ \\
\hline
\end{tabular}

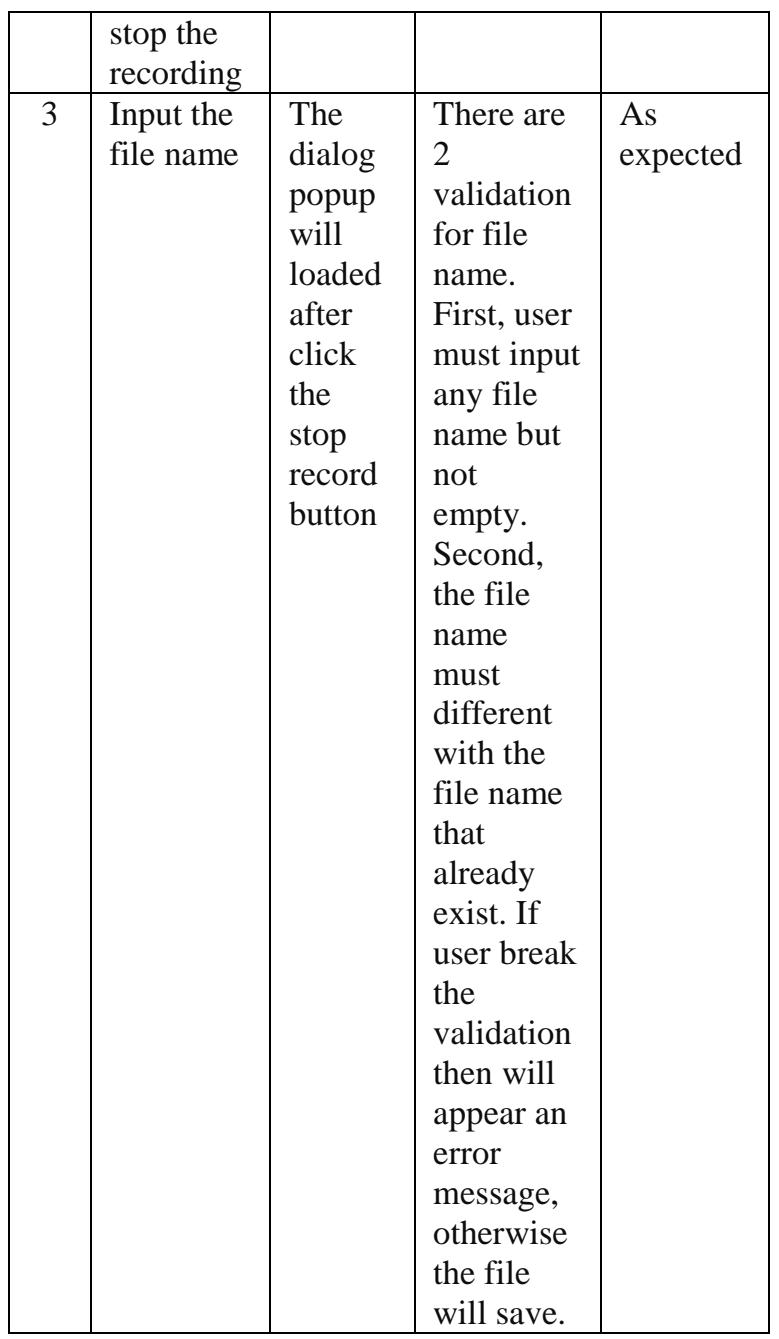

- Open Recorded Music Handling Section

\begin{tabular}{|c|l|l|l|l|}
\hline $\begin{array}{l}\text { N } \\
\text { o }\end{array}$ & $\begin{array}{c}\text { Case } \\
\text { Scenario }\end{array}$ & $\begin{array}{l}\text { Case } \\
\text { Detail }\end{array}$ & \multicolumn{1}{|c|}{$\begin{array}{c}\text { Expected } \\
\text { Result }\end{array}$} & Result \\
\hline 1 & $\begin{array}{l}\text { Open } \\
\text { save } \\
\text { record } \\
\text { music }\end{array}$ & $\begin{array}{l}\text { Click } \\
\text { the } \\
\text { open } \\
\text { save } \\
\text { record } \\
\text { music } \\
\text { button }\end{array}$ & $\begin{array}{l}\text { Open Save } \\
\text { Record } \\
\text { Music is } \\
\text { loaded }\end{array}$ & $\begin{array}{l}\text { As } \\
\text { expecte } \\
\text { d }\end{array}$ \\
\hline 2 & $\begin{array}{l}\text { Playbac } \\
\text { k the } \\
\text { Save } \\
\text { record } \\
\text { music }\end{array}$ & $\begin{array}{l}\text { Click } \\
\text { the } \\
\text { file } \\
\text { name } \\
\text { on list } \\
\text { view }\end{array}$ & $\begin{array}{l}\text { The Music is } \\
\text { start playing }\end{array}$ & $\begin{array}{l}\text { As } \\
\text { expecte } \\
\text { d }\end{array}$ \\
\hline 3 & $\begin{array}{l}\text { Delete } \\
\text { the save } \\
\text { record } \\
\text { music } \\
\text { file }\end{array}$ & $\begin{array}{l}\text { Click } \\
\text { and } \\
\text { hold } \\
\text { the } \\
\text { file } \\
\text { name } \\
\text { for } \\
\text { severa } \\
\text { 1 } \\
\text { second }\end{array}$ & $\begin{array}{l}\text { After click } \\
\text { and hold for } \\
\text { several } \\
\text { second then } \\
\text { the file will } \\
\text { automaticall } \\
\text { y deleted }\end{array}$ & $\begin{array}{l}\text { As } \\
\text { expecte } \\
\text { d }\end{array}$ \\
\\
\end{tabular}


IT FOR SOCIETY, Vol. 03, No. 02

ISSN 2503-2224

- Change Board Handling Section

To make sure if change board feature w

\begin{tabular}{|c|l|l|l|l|}
\hline No & $\begin{array}{c}\text { Case } \\
\text { Scenario }\end{array}$ & $\begin{array}{c}\text { Case } \\
\text { Detail }\end{array}$ & $\begin{array}{l}\text { Expected } \\
\text { Result }\end{array}$ & Result \\
\hline 1 & $\begin{array}{l}\text { Change } \\
\text { the board }\end{array}$ & $\begin{array}{l}\text { Click } \\
\text { one of } \\
\text { Change } \\
\text { board } \\
\text { button }\end{array}$ & $\begin{array}{l}\text { It will } \\
\text { loaded the } \\
\text { board } \\
\text { according } \\
\text { to the } \\
\text { board } \\
\text { chosen }\end{array}$ & $\begin{array}{l}\text { As } \\
\text { expected }\end{array}$ \\
\hline
\end{tabular}

\section{CONCLUSION}

The "Beat Out" application has manage to achieve the research objective, which is able to work as EDM Launchpad composer that contain the Indonesia traditional music source. With a purpose that this application can help user easier to explore their creativities to create EDM using their smartphone in anytime and anywhere.

Here are some of the main features and the advantages of "Beat Out":

1. Beat Out provide 4 different boards. Each board contain 4 loop sounds, 16 beat and melody sounds and 4 traditional music instrument. Therefore there are total 96 sounds that user can choose freely to compose their own EDM.

2. It also come with a feature that helps the user to input their own sound. There are 16 free spaces for user to input their own sound.

3. There is also a feature that helps user to record their own music and plays it again for comparison.

4. This application contain of 5 menus: About, Reset, Traditional Sound Info and Guide.

5. Simplicity, user friendly and interesting UI is one of the good advantages of this application, which let the users to operate the features comfortably

\section{REFERENCES}

[1] Rapid Application Development Model, 2012. Retrieved from http://javatechig.com/se-concepts/rapid-applicationdevelopment-model

[2] Anonymous, Android. https://en.wikipedia.org/wiki/Android_\%28operating_syste m\%29

[3] Wei, Jason. (2012). Android Database Programming. Birmingham: Packt Publishing.

[4] SoundPool. oper.android.com/reference/android/media/SoundPool.html

[5] Anonymous, Electronic Dance Music. https://en.wikipedia.org/wiki/Electronic_dance_music

[6] What is Techno Music? http://www.wisegeek.com/what-istechno-music.htm

[7] Ben Norman, House Music. http://dancemusic.about.com/od/house/g/House_Music.htm
[8] Ben Norman, Trance Music http://dancemusic.about.com/od/genres/g/Trance_Music.ht $\mathrm{m}$

[9] Hardstyle. https://en.wikipedia.org/wiki/Hardstyle

[10] Ben Norman, What is Dubstep. http://dancemusic.about.com/od/genres/g/Dubstep_Definiti on.htm

[11] What is Trap Music? http://runthetrap.com/what-is-trapmusic/

[12] Difference between a MIXER, and a CDJ? https://forums.pioneerdj.com/hc/enus/community/posts/203035119-Difference-between-aMIXER-and-a-CDJ-

[13] Adipro, ALAT-ALAT DJ \& PENGERTIANNYA, http://mixingskill.com/tokoalatdj/index.php?route=pavblog/ blog\&id=9

[14] Download EDM Sound, https://www.freesound.org/

[15] Download Sound Effect, http://www.freesfx.co.uk/

[16] Electronic Sound, http://www.audiomicro.com/free-soundeffects

[17] Instrument Musik Traditional Free,

[18] http://www.stafaband.co/mp3/download/instrumen_musik_t radisional.html

[19] Tradisional Musik Indonesia,

[20] http://mp3take.co/mp3/instrument_musik_indon esia_tradisional.htm 